\title{
ATRIBUCIONES SOBRE EL USO DE LA FUERZA POLICIAL DESDE LA
} PERSPECTIVA DEL AGENTE

\author{
ATRIBUIÇÕES SOBRE O USO DA FORÇA POLICIAL A PARTIR DA \\ PERSPECTIVA DO AGENTE POLICIAL \\ ATTRIBUTIONS OF POLICE USE OF FORCE FROM AGENT PERSPECTIVE
}

\author{
Julián Martínez \\ Facultad Latinoamericana de Ciencias Sociales, Quito, Ecuador
}

Patricia Mariel Sorribas

Universidad Nacional de Córdoba y Universidad Católica de Córdoba, Córdoba, Argentina

\begin{abstract}
RESUMEN
Se describen los contenidos de los procesos atribucionales asociados al uso de la fuerza en agentes de la Policía de la Provincia de Córdoba, Argentina. Los antecedentes indican que, además de los marcos legales, existen sistemas de creencias, valores, actitudes, definiciones morales, aspectos situacionales y una cultura institucional, que operan en los policías al momento de usar la fuerza. Mediante entrevistas en profundidad a 10 agentes policiales de la Provincia de Córdoba, hallamos dimensiones atributivas externas e internas, que a su vez guardan relación con el tipo de trabajo policial según éste sea bajo órdenes o sin ellas. También identificamos relaciones entre dichas dimensiones y diferentes niveles de fuerza que se aplican. Esta investigación cobra importancia por su abordaje psicosocial que prioriza la comprensión de las dimensiones subjetivas de los agentes, evitando explicar la práctica policial únicamente como un mecanismo para el control del orden social y político.
\end{abstract}

Palabras Clave: atribuciones causales; policía; uso de la fuerza; coerción.

\section{RESUMO}

Descrevem-se os conteúdos dos processos atribuicionais associados ao uso da força em agentes da polícia da província de Córdoba, Argentina. Os registros indicam que, além dos marcos legais, existem sistemas de crenças, valores, atitudes, definições morais, aspectos situacionais e uma cultura institucional, presentes na polícia no momento de utilizar a força. Mediante entrevistas em profundidade a 10 agentes policiais da província de Córdoba, achamos dimensões atributivas externas e internas, que guardam relação com o tipo de trabalho policial, segundo este seja sob ordens ou sem elas. Também identificamos relações entre essas dimensões e diferentes níveis de força aplicada. Esta pesquisa torna-se importante por sua abordagem psicossocial, que prioriza a compreensão das dimensões subjetivas dos agentes policiais, evitando explicar a prática da polícia somente como um mecanismo de controle da ordem social e política.

Palavras-chave: atribuições causais; polícia; uso da força; coerção.

\begin{abstract}
This paper describes the contents of attributional processes associated with the use of force by police officers in the province of Córdoba, Argentina. Previous studies point out that in addition to the legal frameworks, there are belief systems, values, attitudes, moral and situational aspects, and an institutional culture that operate in agents when applying coercion. Through interviews with ten officers, we found external and internal attributive factors, which in turn are related to different types of police work: under orders or without them. We also identify linkages between these factors and different levels of force that agents apply. This research becomes important due to the psychosocial approach in which we prioritize understanding of subjective perspectives of officers, avoiding explanations of police practice just as a mechanism to social and political order.
\end{abstract}

Keywords: causal attribution; police; use of force; coercion. 
Desde varias disciplinas se ha estudiado a la policía y los fenómenos asociados a ella. Principalmente se han efectuado aportes desde la sociología de las ocupaciones y organizaciones, criminología, ciencia política, antropología e historia. En América Latina algunos acercamientos han sido planteados a modo de denuncia contra prácticas significadas como abusos de los agentes en casos de violencia y corrupción policial, y otros se han referido a posibles líneas de reforma institucional en contextos de modernización y democratización de los Estados. Sin embargo, son menos los estudios basados en la perspectiva de los agentes policiales sobre sus prácticas.

Estudiar la policía dialogando con sus actores y conociendo de primera mano sus perspectivas apunta a una comprensión de la práctica policial que pretende desmitificar una visión de la institución policial como un aparato represivo del poder estatal (Sirimarco, 2010). En este sentido, intentamos aportar visiones alternativas a las perspectivas normativas y formales de las funciones de la institución policial, a la vez que nos alejamos de los enfoques estigmatizantes de la policía como aparato represivo y corruptivo.

El uso de la fuerza definido como "el uso efectivo o la inminente amenaza del uso de cualquier forma de coacción o incapacitación física contra un ciudadano" (Birkbeck \& Gabaldón, 2002, p. 127), es un fenómeno afectado, en principio, por contextos legales (códigos penal, civil, de procedimiento, etc.), e institucionales (órdenes de los superiores, reglamento interno, etc.). Sin embargo, otros autores (Terrill, Paoline, \& Manning, 2003) advierten que en las intervenciones policiales también están presentes factores de tipo personal y situacional. Por ello, intentamos comprender desde el campo de la Psicología cómo conciben y explican los policías la utilización de la fuerza en encuentros con ciudadanos, y cómo estas concepciones y explicaciones forman parte de su constante construcción y reconstrucción de la realidad.

Las explicaciones sobre el uso de la fuerza se integran en las visiones de mundo de los policías (Suárez de Garay, 2005), incluyendo nociones sobre autoridad, ciudadanía, justicia, etc. En esta construcción de la realidad sus acciones cobran sentidos particulares e intersubjetivos. Por ello, sus explicaciones sobre el uso de la fuerza se abordan desde la Teoría de las Atribuciones Causales (Heider, 1958; Jones \& Davis, 1965; Kelley, 1967), que permite captar procesos interpretativos sobre las causas de un evento o acción, y relacionarlos con el comportamiento de los sujetos (Vizdome-Lozano \& Luciano, 2006). Así las atribuciones sobre el uso de la fuerza policial, constituyen procesos explicativos e interpretativos que realizan los agentes policiales sobre las diversas acciones que implican uso de la fuerza física.

\section{Cultura policial y uso de la fuerza}

La literatura sobre el uso de la fuerza policial ha pasado de centrarse en casos violentos, a intentar comprender el fenómeno desde el punto de vista del trabajo cotidiano de los policías. A nivel local, en la Provincia de Córdoba, Argentina, pocas investigaciones empíricas se han ocupado del tema. Algunas se orientaron hacia una crítica de la institución (por ejemplo: Seveso \& Cabral, $2009^{1}$ ) analizando casos específicos significados como abusos policiales a partir de denuncias de los ciudadanos. En cambio, Hathazy (2006), desde un análisis simbólico de la experiencia corporal, aborda los significados y sentidos que los Policías Antidisturbios le dan al uso de la fuerza, concluyendo que mediante la adquisición de aptitudes como coraje, "temple" y masculinidad en las instancias formativas, aquélla deviene fuente de identidad, sentido y disfrute.

Por otra parte, los aportes provenientes de otros contextos han sido recopilados por McCluskey, Terrill y Paoline (2005), quienes identificaron tres principales perspectivas de análisis: (a) la influencia de la organización, (b) los aspectos situacionales, y (c) las características personales del policía. El enfoque de la influencia organizacional sostiene que los policías encaran colectivamente tensiones laborales, y por ello también comparten actitudes y valores orientados hacia ciudadanos, jefes, supervisores y procedimientos (Terrill et al., 2003). Este set de valores y actitudes funciona como una mediación entre las normas formales y las prácticas concretas de los agentes, configurando una Cultura Policial (Terrill et al., 2003) o una Cultura Profesional del policía (Yñiguez Navas, 2007).

En este sentido Terrill et al. (2003) a partir de una investigación comparativa de dos departamentos de policía de diferentes localidades en Estados Unidos, sostienen que los policías alineados con una cultura policial, que los autores denominan "tradicional", emplearán en mayor medida mecanismos coercitivos en su labor. La cultura policial tradicional se caracteriza por "actitudes desfavorables hacia los ciudadanos y supervisores, desdén hacia las pautas de procedimiento, rechazo a los roles que no impliquen combate a la delincuencia, valoración positiva del patrullaje agresivo, y selectividad en la aplicación de la ley" (Terrill et al., 2003, p. 1007).

Por su parte, analizando elementos de la cultura policial mexicana, Suarez de Garay (2005) señala que al 
interior de la institución policial los agentes se adaptan re-conociendo y re-produciendo habilidades, normas y valores con el fin de lograr reconocimiento e identidad. Allí alimentan mecanismos de aislamiento social, lealtad grupal y autorreferencia que posteriormente enmarcarán las interacciones con los ciudadanos. Uno de los efectos es el uso de un lenguaje eufemístico, de carácter principalmente judicial, que permite a los policías la construcción de un entorno simbólico en el cual interpretan y explican sus prácticas, y que marca diferencias entre el "nosotros" policial y el resto de la sociedad (Azaola \& Ruiz, 2010).

Sin embargo, la referencia a la cultura policial como marco general de explicación de las prácticas de los agentes ha sido objeto de críticas. Distintos tipos de organización, con distintos tipos de funcionamiento (por ejemplo: legalista, de vigilancia, o de servicio) implicarían diferencias en la cultura organizacional y por ende en el uso de la fuerza de los policías (Westley, 1970, citado por McCluskey et al., 2005). Lo mismo se puede aplicar para diferentes unidades al interior de una misma institución policial (por ejemplo: unidades antidisturbios, policía preventiva, tránsito, etc.). Además Yñiguez Navas (2007) sostiene que las respuestas de los policías, dependen del conocimiento de las situaciones y están limitadas a las posibilidades de decisión en cada una de ellas.

Es así que el enfoque situacional estudia el uso de la fuerza como respuesta a interacciones condicionadas por factores contextuales específicos. Estos factores están constituidos principalmente por: (a) la categorización de los ciudadanos que realizan los policías en base a su género, edad, pertenencia etno-racial y estatus social; y (b) el comportamiento de los ciudadanos, como resistir a los requerimientos del oficial, agredirlo, faltarle al respeto, o evidenciar alteración o escaso autocontrol por ingesta de alcohol o drogas (Terrill et al., 2003).

Evaluar las características y el comportamiento de los ciudadanos permite a los policías desarrollar tácticas para utilizar la fuerza basados en juicios sobre su estima moral y capacidad de reclamo (Birkbeck \& Gabaldón, 2002). Así, identificamos una dimensión moral del uso de la fuerza vinculada al enfoque situacional. De hecho, Garriga (2010) afirma que los policías realizan definiciones morales de las situaciones, las que los habilitan, o no, a usar la fuerza. Para los policías el uso de la fuerza sería una respuesta moralmente tolerable en determinada situación, a lo cual Garriga (2010) denomina réplica, usando un término nativo.

La dimensión moral alienta también preguntas sobre las diferencias interindividuales en el uso de la fuerza: ¿Todos los policías usan la fuerza de la misma forma en situaciones similares? ¿Un policía emplea un mismo esquema moral en situaciones diferentes?

El enfoque individual se ha ocupado de relacionar características individuales de los policías (valores, actitudes, experiencias, puntos de vista, educación y capacitación) con el uso de la fuerza (McCluskey et al., 2005; Terrill \& Mastrofski, 2002). Desde esta perspectiva, autores como Worden (1995) y Monjardet (2010) han elaborado tipologías a partir del análisis de actitudes y valores de los policías. Sin embargo, estos mismos elementos considerados a nivel agregado por otros autores (Terrill et al., 2003), han sido la base de la conceptualización de una cultura policial.

Este recorrido nos indica que debemos incluir en nuestro análisis factores institucionales, situacionales, e individuales. El enfoque de la Teoría de las Atribuciones Causales posibilita la indagación de esos factores mediante el relevamiento de las explicaciones de los policías sobre el uso de la fuerza. Además de ello, las diferentes perspectivas revisadas resultan útiles para organizar en categorías los diferentes tipos de atribuciones que los policías realizan.

\section{La perspectiva atribucional}

Estudiar el uso de la fuerza policial a partir del marco de las atribuciones causales tiene relevancia a nivel cognitivo y comportamental. La Teoría atribucional nos ayuda a responder a la pregunta sobre las causas del comportamiento en términos de procesos interpretativos (Jones \& Davis, 1972, citado por Hewstone, 1992; Vizdome-Lozano \& Luciano, 2006). Las atribuciones causales ayudan al control cognitivo de la realidad y se relacionan con la toma de decisiones, elaboración de juicios y refuerzo de convicciones, e intervienen mediando entre percepción e interacción social a nivel individual y grupal (Hewstone, 1992). Para Richaud de Minzi (2004) lo nuclear de la teoría de la atribución reside en que el constructo reduce la brecha entre la información que proviene del mundo exterior y el significado que le atribuye el sujeto. Por esto mismo no sería un proceso más cognitivo que afectivo o motivacionalmente orientado.

Para comprender la estructura de la causalidad que perciben los actores, una primera dimensión analítica distingue entre factores causales internos y externos al sujeto. Es decir, las personas identifican causas que refieren a sus características personales o a características externas, de la tarea o la suerte (Hewstone, 1992). Cabe aquí diferenciar la dimensión externo-interno de las causas disposicionales y 
situacionales. Un observador puede atribuir la causa del comportamiento de otra persona a características personales, lo que constituye una atribución disposicional, mientras que puede atribuir su propio comportamiento a elementos de la situación, constituyendo una atribución situacional (Jones \& Nisbett, 1972, citado por Vásquez Cabrera, 2008). Es decir, pueden existir atribuciones externas de tipo disposicional o situacional.

En la práctica, factores externos e internos no son excluyentes, y cuando las personas realizan atribuciones con sus propias palabras recurren tanto a factores internos como externos (Lalljee, 1982), con la finalidad de lograr precisión atributiva (Hewstone, 1992). Sumado a la dimensión del locus (interno - externo), Weiner (1985a, citado por Hewstone, 1992) ha propuesto complementar la estructura de las atribuciones causales agregando las dimensiones de estabilidad que se refiere a si la causa se mantiene en el tiempo, y controlabilidad, referida al control o influencia que el sujeto puede tener sobre las mismas.

Empíricamente los procesos atribucionales se han estudiado a nivel individual e intergrupal. Hewstone (1992) señala que entre grupos, las atribuciones cumplen la función de mantener la imagen e identidad social positiva. Para ello, por ejemplo, los grupos atribuyen sus actos positivos a causas internas, estables, controlables y sus actos negativos a causas externas, y/o poco controlables. Asimismo, Tajfel (1969, citado por Hewstone, 1992) señala que las atribuciones intergrupales cumplen una función de simplificación del entorno cognitivo. Por ello, consideramos que el análisis intergrupal de los procesos atribucionales enriquece el estudio del uso de la fuerza policial, ya que ésta ocurre entre individuos con identidades sociales consolidadas, y según algunos autores, está mediada por categorizaciones sociales que los policías realizan sobre los ciudadanos. En términos de la teoría de las atribuciones, las personas atribuyen comportamientos según la pertenencia grupal (Hewstone, 1992), es decir, con referencia a un grupo, las personas explican el comportamiento y ubican socialmente a los otros.

Los estudios atribucionales sobre acciones violentas han hecho mayor énfasis en el análisis de casos de terrorismo y violencia política (Manzi, Ruiz, Krause, \& Meneses, 2004; Pronin, Kennedy, \& Butsch, 2006; Sabucedo, Blanco, \& De la Corte, 2003; Vásquez Cabrera, 2008) y sobre acciones violentas en la familia o parejas (Lozano Gerena, Castro Guerrero, \& Moreno, 2008; Marrero, Moraza Pulla, Matud Aznar, Carballeira Abella, \& Aguilera Ávila, 2003) y han aportado sustancialmente al conocimiento de las causas percibidas por los actores (afectados o no) sobre dichos fenómenos. Sin embargo, no hallamos estudios empíricos que hayan abordado, desde la perspectiva atribucional y específicamente en la población policial, el fenómeno del uso de la fuerza. Es allí donde encontramos un espacio de investigación desde el cual podemos contribuir a los estudios policiales y atribucionales desde un enfoque psicosocial.

\section{Metodología}

Utilizamos un enfoque cualitativo y un diseño flexible para caracterizar las dimensiones atribucionales sobre el uso de la fuerza y la interconexión entre las mismas. La comparación constante (Glaser \& Strauss, 1967), y la revisión teórica orientaron el relevamiento, la categorización y el ordenamiento de los datos, tratando que conceptos y categorías se ajusten a ellos.

Mediante un muestreo teórico accedimos a diez policías de la Provincia de Córdoba en servicio activo, cuatro mujeres y seis varones. El participante de menor edad tenía 26 años de edad y dos años de antigüedad en la fuerza al momento de la entrevista, y el mayor tenía 46 años y 24 años en la institución. La selección de los casos fue realizada simultáneamente con la codificación y análisis. Esta estrategia se llevó a cabo hasta que consideramos que no se hallaba información adicional que permitiera desarrollar nuevas propiedades o variaciones en las categorías.

Realizamos entrevistas en profundidad que tuvieron una duración promedio de 45 minutos. La guía de entrevista se basó en los objetivos de la investigación y en la revisión de antecedentes. No existía una secuencia fija de preguntas sino más bien una agenda conversacional que marcaba el terreno narrativo (Holstein \& Gubrium, 1995). Este instrumento nos permitió abordar los temas de interés con un margen de flexibilidad (Pinto Mascareño, 2010), y formular repreguntas atendiendo a la interacción con los participantes, en búsqueda de nuevas categorías.

Las preguntas iniciales intentaban generar un clima de confianza y reducir la ansiedad generada por la situación de entrevista. Indagamos aspectos generales de los participantes (edad, antigüedad), motivos para ingresar a la institución, y otros referidos a su cotidianeidad laboral. Posteriormente, preguntamos sobre situaciones en las que podría darse el uso de la fuerza (cacheos, arrestos, reducciones, uso de armas, etc.), tratando que los participantes nos den algo más que definiciones normativas o puramente legales, e intentando rescatar su perspectiva a partir de sus experiencias (Holstein \& Gubrium, 1995). 
Las entrevistas, que fueron registradas digitalmente, contaron con el consentimiento de los participantes. Formulamos las preguntas de modo que no resultaran amenazantes ni expresaran un sesgo (Festinger \& Katz, 1992). Sin embargo, en algunos casos fue evidente la presencia de respuestas lacónicas y estereotipadas, referidas principalmente a explicaciones normativas. Interpretamos que los participantes pudieron pensar que la expresión de sus opiniones pudiera ser peligrosa y causar represalias al interior de la institución, y también que el uso del grabador pudo haber condicionado las respuestas de los sujetos. En aquellos casos, tras apagar el grabador, las entrevistas continuaron proporcionando datos significativos que fueron registrados como notas de campo.

Accedimos a los participantes mediante el contacto con personas que tuvieran algún vínculo familiar o de amistad con policías. Esta recomendación fue una estrategia que generó una mejor disposición de los agentes durante la entrevista. Las entrevistas finalizaban solicitando el contacto con otros compañeros que quisieran realizar la entrevista, lo cual fue fructífero para obtener más casos, y los siguientes participantes tenían mejor disposición al ser recomendados por sus compañeros. La mayor parte de las entrevistas fueron realizadas en los domicilios de los participantes, exceptuando dos que se realizaron en comisarías y una en el lugar de trabajo donde la agente se encontraba realizando 'adicionales' ${ }^{2}$.

A medida que avanzábamos con las entrevistas pudimos ajustar nuestro lenguaje para hacerlo más familiar a los participantes. Esto resultó en la adopción de ciertas expresiones y la comprensión de los sentidos que ellos le otorgaban a las palabras. Esto también nos facilitó la conversación y el entendimiento de las perspectivas de los agentes.

Se han tenido en cuenta las consideraciones éticas sobre consentimiento informado, anonimato de los participantes y confidencialidad de los datos. Todos los participantes fueron consultados sobre su participación en la investigación, aclarando que podían retirarse de la misma si lo desearan. Los nombres de los participantes han sido modificados atendiendo a dichas consideraciones.

\section{Análisis de datos}

Utilizando el método de comparación constante (Glaser \& Strauss, 1967), partimos de la definición de uso de la fuerza señalada anteriormente, y codificamos como unidades de registro los fragmentos que contenían expresiones donde se hacía referencia al contacto físico con ciudadanos. Teniendo en cuenta el carácter eufemístico del lenguaje policial, utilizamos un criterio semántico para la codificación que permitió la inclusión de expresiones como: 'procedimientos', 'reducciones', 'aprehensiones', 'trasladar', etc., que implicaban uso de la fuerza. Como unidades de contexto se tuvieron en cuenta los elementos atributivos de las expresiones sobre el uso de la fuerza.

Mediante codificación axial identificamos propiedades que permitieron clasificar las atribuciones de los agentes sobre el uso de la fuerza en diferentes categorías. Se tuvieron en cuenta también casos negativos (de no uso, o aplazamiento del uso de la fuerza) y casos únicos, donde un solo incidente pudo ser suficiente para producir una categoría conceptual (Glaser \& Strauss, 1967).

De esta manera descubrimos que las atribuciones sobre el uso de la fuerza en el trabajo policial se caracterizan por referirse a factores externos (situacionales y disposicionales), y a factores internos de los policías. Asimismo, hallamos que el contenido de las atribuciones difiere en función de dos tipos principales de encuadres del trabajo policial: (a) con órdenes, más heterónomo, y (b) sin órdenes, más autónomo. Finalmente pudimos notar la existencia de varios niveles de fuerza que se aplican incrementalmente y son atribuidos a combinaciones de factores externos e internos. Cabe señalar que la categorización atiende a una necesidad analítica y descriptiva, pero los participantes generalmente elaboran más de una atribución para una misma circunstancia, dando explicaciones multicausales. Es decir, que algunas explicaciones de los agentes se refieren simultáneamente a varios factores atribucionales.

Evidentemente, las limitaciones de tiempo y recursos, por un lado, y del acceso a la muestra por el otro, han limitado en cierta medida el número de casos. Sin embargo, hemos tomado en cuenta los criterios de credibilidad, transferibilidad, seguridad y confirmabilidad, propuestos por Guba y Lincoln (1985, citado por Mendizábal, 2006) para garantizar calidad metodológica en investigaciones cualitativas. Para ello, realizamos un registro responsable, preciso y rico en detalles, sin alterar en ninguna forma los testimonios de los entrevistados, ni los significados atribuidos. También explicitamos los procedimientos mediante los cuales otros investigadores pueden tienen la posibilidad de replicar el estudio para contrastarlo en contextos similares. 


\section{Resultados}

Como ya se precisó, identificamos dos modos de acción en el trabajo policial en los cuales hallamos atribuciones externas e internas que explicarían el uso de la fuerza. El trabajo sin órdenes es realizado principalmente en labores de patrullaje y vigilancia, y presenta un mayor grado de autonomía respecto al uso de la fuerza. En el trabajo realizado bajo órdenes se debe cumplir estrictamente lo que manda el superior (heteronomía). Ello implica que la estructura causal sobre el uso de la fuerza en cada tipo de trabajo también presenta algunas diferencias.

\section{Actuando con órdenes}

Aquí se analizan explicaciones referidas a dos tipos de intervenciones policiales que, sin ser equiparables, comparten el rasgo de estar mediadas por órdenes que los agentes deben cumplir: Guardia de Infantería y Órdenes Judiciales. El trabajo en Guardia de Infantería consiste en el "mantenimiento del orden, mediante la amenaza o el uso de la fuerza física", interviniendo principalmente en "situaciones multitudinarias como manifestaciones, partidos de fútbol y conciertos" (Hathazy, 2003, p. 39). Las intervenciones de Guardia de Infantería las realizan "grupos de combate" equipados con elementos especiales de defensa y ataque (cascos, escudos, gas, escopeta con proyectiles de goma, bastones). Los grupos de combate se componen generalmente de ocho policías con funciones específicamente asignadas y sus acciones están bajo las órdenes de un jefe del grupo, el cual a su vez está bajo el mando del jefe de todo el operativo.

Las atribuciones externas respecto del uso de la fuerza en este tipo de intervención refieren a la interacción con el público y a las órdenes de los jefes. El comportamiento del público, según los policías, determina el incremento del uso de la fuerza. La agresividad y la intención de confrontar son atribuidas a un grupo menor del público, los "agitadores", que se diferencian del resto de los "manifestantes". Así, según la clasificación y comportamiento del público, los policías explican que se pasa de roles pasivos como "aguantar" las agresiones de los manifestantes, a estrategias más activas como avanzar en el terreno demostrando el potencial de fuerza y disuadiendo al público. $\mathrm{Si}$ estas acciones no son suficientes, se incrementan los niveles de fuerza con acciones represivas que incluyen el empleo de bastones, agua, gas, proyectiles de goma, y finalmente proyectiles de metal.
En el trabajo de Guardia de Infantería los policías no pueden actuar por su cuenta y explican que la aplicación de mayores niveles de fuerza depende de la disposición explícita del jefe de los operativos. Uno de los entrevistados, Pablo, aclara que ellos están " $a$ las órdenes del jefe de grupo que ... es quien dispone si vos procedes [a usar la fuerza] o no" " ${ }^{3}$ Estas órdenes superiores a veces son interpretadas por los policías como un límite al despliegue de su total potencial de fuerza, y vistas como un factor ego-protectivo de los jefes para evitar sanciones institucionales y sociales por las posibles repercusiones del uso de la fuerza en forma de represión.

Las atribuciones internas en el trabajo de Guardia de Infantería refieren tanto a la capacitación, preparación y experiencia de los agentes adquiridas en instancias de formación, como al cansancio y estrés que sufren en el desempeño de sus labores. La capacitación y la experiencia, en palabras de otro participante, Mauricio, les permiten "saber mucho mejor, tener mucha más práctica [sobre lo] que tenés que hacer en determinados momentos". Asimismo, las atribuciones referidas al estrés y cansancio, indican que dichos factores operarían haciendo que en ocasiones se cometan "errores" o "excesos" al usar la fuerza. Estas atribuciones son relevantes a nivel explicativo y justificativo para los policías, quienes enfatizan que sus jornadas laborales habitualmente exceden las 24 horas consecutivas. Si bien el estrés o cansancio pueden ser atribuidos a la dificultad de la tarea, los hemos considerado factores internos porque se refieren a determinantes internos de los agentes sobre el uso de la fuerza.

Los agentes de infantería señalan que al momento de utilizar la fuerza, los valores como el autocontrol o el "temple" se convierten en símbolos de superioridad y distinción. La disciplina y el sacrificio los distinguen; y aunque podrían desplegar todo su potencial de fuerza, su mayor mérito y demostración de supremacía consiste en no verse alterados por las afrentas del público. Hernán, otro participante, cuenta que "ellos [los manifestantes] te agreden, te buscan la reacción ... Pero el policía tiene una característica que aprendió en el campo de instrucción, el policía está ejercitado en la humillación ... Difícilmente me puedan humillar en una manifestación, muy difícil". Se ve entonces claramente un atributo disposicional interno capaz de modular el comportamiento del policía, y la atribución de características violentas se perciben como intrínsecas al grupo con el que interactúa.

En cuanto al cumplimiento de órdenes judiciales existe una clara diferencia respecto al tipo anterior. $\mathrm{La}$ 
organización, planificación y ejecución de las acciones que realizan los policías cuando están cumpliendo una orden de exclusión o allanamiento deja un margen de decisión más amplio a los agentes en comparación con las órdenes de Guardia de Infantería.

Los factores externos más importantes refieren a la existencia misma de una orden proveniente del sistema de justicia que los policías están obligados a cumplir. Silvia explica que ante una orden judicial "nosotros tenemos que acatar esa orden". También identificamos como factor externo el comportamiento de los ciudadanos, algunas veces atribuido a grupos de referencia, a partir de categorizaciones que realizan los policías. Esto último es similar tanto en el trabajo bajo órdenes como sin ellas, por lo que lo detallaremos en el siguiente apartado. Lo característico del cumplimiento de órdenes judiciales es que el margen de acción es más amplio, por lo que las habilidades individuales de los agentes para usar la fuerza cobran importancia. Así, la "experiencia" o "el criterio" para usar la fuerza son factores internos que denotan el reconocimiento de la habilidad para interpretar las situaciones y actuar en consecuencia con esas interpretaciones. En las explicaciones se combinan las "lecturas" (con mayor o menor experiencia o criterio -factores internos-) que se hagan de las situaciones (comportamiento de los ciudadanos -factores externos-) para definir el trato con el otro, es decir, si se aplica mayor o menor fuerza.

\section{Actuando sin órdenes}

La característica de este tipo de acciones es que no están mediadas por órdenes específicas provenientes de mandos superiores o de la justicia, sino que las intervenciones responden a la obligación de la policía de atender casos de flagrancia o al llamado de un ciudadano. Los factores externos refieren a: (a) el comportamiento del ciudadano, (b) la categorización del ciudadano, (c) las exigencias institucionales, (d) el peligro percibido en la situación, y (e) las definiciones morales de las situaciones.

En aquellas atribuciones referidas al comportamiento de los ciudadanos hallamos en primer lugar la constatación de un delito o una contravención, donde el uso de la fuerza es explicado como forma de impedir o detener el delito. Sin embargo, el caso de las contravenciones es más complejo porque en la Provincia de Córdoba está en vigencia la Ley 8.431 que estipula un Régimen de Contravenciones o "Código de Faltas" en el que se tipifican sanciones sobre infracciones de los ciudadanos (Ley n. 8431, 2007). Algunos de los artículos y procedimientos allí establecidos son imprecisos y en ciertos casos entran en contradicción con derechos establecidos en la Constitución Nacional Argentina ${ }^{4}$. El artículo 98 referido al "merodeo" es el más conflictivo. Emilse explica qué sucede en una detención por merodeo: "si vos pasás y ves que están espiando una casa, eso ya es una actitud sospechosa. ... Y ahí en el código de faltas nuestro tenemos el merodeo, entonces los llevamos por eso". La iniciativa de la acción corresponde al agente y se hace explícita una categorización social del ciudadano y una identidad grupal fuerte: "nuestro".

Otro atributo causal externo para el uso de la fuerza es la resistencia del ciudadano, es decir cuando se rehúsa a cumplir los requerimientos del policía (por ejemplo: negarse a un registro o cacheo, negarse a ingresar a la patrulla). En los casos de resistencia se utiliza mayor fuerza porque la mediación de las palabras deja de ser efectiva. José explica que "si ya no lo convences ahí se usa la fuerza”. Hernán es más enfático al respecto: "No te queda otra. Fuerza! [A] un hombre que se resistió, ... lo agarré del cogote, lo tiré a la vereda, le puse una pata encima". La agresión al agente también constituye un factor explicativo del uso de la fuerza: "si el tipo empieza a usar la fuerza primero... bueno, yo no le voy a leer los derechos, no" relata Hernán.

Relacionado con la conducta de los ciudadanos, pero no restringido a ella, para los policías existen situaciones que son percibidas como riesgosas, ante las cuales justifican el uso de la fuerza como una forma de preservar su vida y la de terceros. Es el caso de enfrentamientos que involucran disparos o explosivos, o casos en que los ciudadanos portan armas. Al hallarse ante una situación riesgosa, los policías explican que su predisposición al uso de elevados niveles de fuerza es mayor, ya que está en juego su vida, la de sus compañeros o la de otros ciudadanos.

Pero los policías no emplean la fuerza con todos los ciudadanos por igual. Las categorizaciones que realizan están implicadas en el contenido que asumen sus explicaciones. Hallamos que para los policías existen básicamente tres tipos de ciudadanos: (a) "Los choros" "ante quienes el uso de la fuerza se justifica porque poseen determinadas características (rasgos físicos, vestimenta, lenguaje) estables en el tiempo, que además son compartidas por otros ciudadanos categorizados también dentro del mismo grupo. Compartir dichas características implica automáticamente una pertenencia social, lo cual deviene factor explicativo en las atribuciones de los policías; (b) "Los borrachos" que son personas a las que se atribuye algún tipo de alteración por ingesta de alcohol o drogas, de menor estabilidad temporal que en el caso anterior. Los policías explican que se utiliza la fuerza para "protegerlos" a ellos mismos y 
a terceros de las posibles consecuencias de su estado y (c) "La gente común" quienes no representan ningún peligro y se les atribuye una identidad social positiva. Cuando éste tipo de ciudadano es objeto del uso de la fuerza por parte de la policía, las explicaciones refieren a estados transitorios de alteración como en el caso anterior, y su accionar no es visto en términos de agencia intencional.

Los policías también explican que existen exigencias de los superiores para realizar detenciones. Esto se convierte en otro factor explicativo de tipo externo que - sin ser "órdenes" en el sentido del apartado anterior - se enmarcan en un sistema intraorganizacional de premios y sanciones sobre los resultados del trabajo policial y permite reconocer el poder atribuido a los jefes como un factor explicativo.

Finalmente para los agentes hay situaciones que no son moralmente tolerables, ante las cuales elevados niveles de fuerza serían la respuesta "adecuada". Diego relata que en una ocasión pudo enfrentar a un sujeto que momentos antes había sido identificado como responsable de violar a un menor: "Se me salió el pibe de barrio y le pegué por donde pude ... lo lastimé y lo lastimé muy mucho". La estructura causal está conformada por las características de una situación externa al policía, pero también por la definición moral que él realiza sobre la misma, por lo que podemos comprender mejor a las definiciones morales del uso de la fuerza como una bisagra entre factores explicativos externos e internos. Por un lado, habría atributos personales que permiten evitar o aplazar el uso de la fuerza, o atributos que permiten la expresión de un uso de la fuerza de carácter más técnico que impediría ocasionar daño al sujeto. Sin embargo, también habría factores que se activarían frente a situaciones extremas que al agente le resultan intolerables moralmente. De allí que su comportamiento sea menos "técnico" y menos eficiente en la capacidad aminorar el daño al otro.

Algunos de los factores internos coinciden con aquellos vistos en el trabajo con órdenes: cansancio por jornadas laborales extensas, estrés y autocontrol son los más importantes. El "criterio" para actuar también se presenta como factor interno relevante debido al mayor margen de acción en el trabajo sin órdenes. Por ello la auto-atribución de capacidad, preparación y sobretodo de un nivel suficiente de experiencia son factores que median en la utilización de la fuerza, generalmente para aplazarla o aminorarla.

Finalmente, se suma la personalidad del agente para utilizar la fuerza, en términos disposicionales. Es llamativo constatar que los policías atribuyen a "otros" la utilización de la fuerza por el disfrute que les produce. Si bien este esquema atribucional se presentó en un único caso, nos permite estimar que la personalidad de cada policía sería un factor explicativo presente, pero que es atribuido a otros por efectos de deseabilidad social. Diego ilustra esto comentando que "hay tipos [policías] que pegan porque les gusta pegar".

Todos estos factores en los diferentes modos de acción se combinan en las explicaciones de los agentes, quienes utilizan diferentes niveles de fuerza que van desde el contacto físico únicamente con técnicas corporales como llaves, golpes, etc., al uso de elementos como esposas o bastón; pasando por el uso del arma de modo persuasivo y finalmente la realización de disparos. Es decir, la combinación de factores, internos y externos, junto al tipo de encuadre del trabajo policial, conforman una estructura multicausal de las explicaciones de los agentes respecto a los diferentes niveles de fuerza que utilizan con diferentes sujetos sociales.

\section{Conclusiones}

La teoría de las atribuciones brinda un nuevo enfoque al estudio del uso de la fuerza policial. Su aporte descansa en la importancia de los procesos atributivos en el comportamiento, particularmente en la toma de decisiones y elaboración de juicios a niveles interindividuales e intergrupales (Hewstone, 1992). Aquí hemos abordado un fenómeno complejo, analizando las reconstrucciones de los agentes de policía sobre sus prácticas. En ellas encontramos, a veces, esquemas explicativos más estandarizados para explicar y explicarse el uso de la fuerza. Tal es el caso de las acciones bajo órdenes. Y otras veces reconocemos explicaciones más ricas y complejas en términos causales, principalmente en acciones "sin órdenes".

Nuestros resultados sugieren que las explicaciones de los policías se caracterizan por su multicausalidad, es decir, por la combinación de varios factores tanto internos como externos, lo que da cuenta de la complejidad de las mediaciones psicosociales que intervienen en una situación donde un policía hace uso de la fuerza. Por ello, podemos decir que las caracterizaciones genéricas de la policía, o de la cultura policial, no son suficientes para explicar el comportamiento de los agentes y dejan escapar estos matices, complejidades y múltiples combinaciones de factores explicativos, que los policías estiman determinantes y que un estudio desde la perspectiva de los procesos atributivos permite reconocer. 
Por otra parte, la perspectiva de los procesos atribucionales nos permitió comprender el uso de la fuerza como un fenómeno asociado a demandas pragmáticas del trabajo policial y a su vez modulado por factores internos de los policías. Por ejemplo, en un caso de flagrancia, donde se constata un robo, los policías explican que se interviene para cesar el delito y aprehender al sospechoso y la utilización de la fuerza es explicada-en principio - por las características de la situación. Sin embargo, al profundizar en la estructura causal se identifican otros factores intervinientes como la categorización del ciudadano basada en su comportamiento, su estima moral, sus rasgos etno-raciales o de clase; factores que, por ejemplo, justificarían la aplicación de mayores niveles de fuerza. En cambio, la consideración de las posibles sanciones derivadas de un exceso, sumado al autocontrol y la experiencia (factores internos), actuarían modulando o atenuando los niveles de fuerza empleados.

También debemos señalar que, respecto a la mayor o menor autonomía del trabajo policial, las exigencias por parte de los superiores abren un campo para futuras investigaciones en las cuales sería interesante profundizar cómo se constituyen las atribuciones de 'poder' a los jefes, lo que hace que las evaluaciones y valoraciones de los propios agentes sean dejadas de lado. Es decir, cómo el poder atribuido al jefe modifica la definición moral del agente sobre lo correcto e incorrecto del uso de la fuerza, menoscabando su autonomía. En ellas se podría analizar cómo son representados los superiores y en qué consiste efectivamente la capacidad de lograr que los policías apliquen la fuerza a pesar de evaluaciones negativas sobre estas prácticas, así como también el desarrollo de mecanismos protectivos para evitar posibles sanciones.

También debemos señalar que una perspectiva del uso de la fuerza que toma en cuenta las relaciones intersubjetivas, no deja de lado los condicionamientos institucionales. Partiendo de la concepción de la policía como el órgano legal y legítimamente habilitado para usar la fuerza, es claro que la institución policial tiene mucha influencia en la conducta de los agentes. Esto no quiere decir, sin embargo, que el condicionamiento institucional sea el único intermediador en el uso de la fuerza. Hemos tratado de mostrar que para cumplir el mandato institucional de mantener el orden y la seguridad del estado, la práctica de los agentes está mediada por otros factores psicosociales, y no solo por las reglas u orientación institucional.

Por ello y para finalizar, queremos señalar que consideramos que el diseño e implementación de políticas de formación policial basadas en el respeto a los derechos humanos debieran tener en cuenta estos procesos psicosociales que median estas interacciones. Los hallazgos referidos a los modos de aplazar o evitar el uso de la fuerza, nos habilitan a pensar que existen diferentes estilos personales de ejercer el rol policial. Las atribuciones referidas a la experiencia, al criterio, o a la personalidad de los policías nos dan pistas para comprender por qué algunos agentes parecieran tener más márgenes para interactuar con los ciudadanos evitando, aplazando o aminorando el uso de la fuerza, y otros no. Esto da cuenta de que el uso de la fuerza no es una interacción inevitable entre actores que se enfrentan, sino que existe un grado de autonomía y atributos personales de cada agente intervienen en su concreción, sobretodo en el trabajo policial "sin órdenes". Podemos entonces aportar al diseño de políticas institucionales que promuevan estos factores con miras a lograr interacciones en las cuales el respeto de los derechos de los ciudadanos esté en primer plano.

\section{Notas}

Seveso, E. \& Cabral, X. (2009). Contra la pared: los cuerpos del delito. Descripción y análisis de algunas escenas de violencia policial. Ponencia presentada al XVII Congreso ALAS: Latinoamérica Interrogada. 31 de agosto al 4 de septiembre de 2009. Universidad de Buenos Aires (UBA).

2 Los "Adicionales" son trabajo extra, no obligatorio, que realizan los policías en lugares donde se solicita presencia policial. Ellos representan un ingreso económico extra para la mayoría de policías.

3 Los nombres de los participantes han sido modificados respetando su anonimato en esta investigación. Los fragmentos de texto que aparecen en cursiva y entre comillas pertenecen a lo dicho por los agentes de policía.

4 Constitución Nacional Argentina. En http://www.senado. gov.ar/Constitucion/capitulo1

5 La palabra "choro" proviene del lunfardo, o lenguaje de uso popular en Argentina y Uruguay, y se utiliza para referirse a un ladrón, o una persona que roba o hurta.

\section{Referencias}

Azaola, E. \& Ruiz, M. (2010). Papeles policiales: abuso de poder y eufemismo punitivo en la policía judicial de la ciudad de México. Desacatos, 33, 95-110.

Birkbeck, C. \& Gabaldon, L. (2002). La disposición de agentes policiales a usar la fuerza contra el ciudadano. In R. Briceño León (Comp.), Violencia, sociedad y justicia en América Latina (pp. 229-243). Buenos Aires: CLACSO.

Festinger, L. \& Katz, D. (1992). Los métodos de investigación en las ciencias sociales. Barcelona: Paidós.

Garriga, J. (2010). "Se lo merecen". Definiciones morales del uso de la fuerza física entre los miembros de la policía bonaerense. Cuadernos de Antropología Social, 32, 75 - 94.

Glaser, B. G. \& Strauss, A. L. (1967). The discovery of grounded theory: Strategies for qualitative research. Chicago: Aldine Publishing Company. 
Hathazy, P. (2003). Fighters of order: Understanding riot police use of force. Master's Thesis, International Institute for the Sociology of Law, Oñati, Espanha.

Hathazy, P. (2006). Los cuerpos del orden. Técnicas corporales y trabajo policial antidisturbios. In V. Papalini (Ed.), $L a$ comunicación como riesgo (pp. 45-78). La Plata: Al Margen.

Heider, F. (1958). The psychology of interpersonal relations. New York: Wiley.

Hewstone, M. (1992). La atribución causal. Del proceso cognitivo a las creencias colectivas. Barcelona: Paidós.

Holstein, J. A. \& Gubrium, J. F. (1995). The active interview. London: SAGE Publications.

Jones, E. E. \& Davis, K. E. (1965). From acts to dispositions: The attribution process in social psychology. In L. Berkowitz (Ed.), Advances in experimental social psychology (Vol. 2, pp. 219-266). New York: Academic Press.

Kelley, H. H. (1967). Attribution theory in social psychology. In D. Levine (Ed.), Nebraska Symposium on Motivation (Vol. 15, pp. 192-238). Lincoln: University of Nebraska Press.

Lalljee, M. (1982). Teoría de la atribución y análisis de las explicaciones. Estudios de Psicología, 12, 47-62.

Ley n. 8431. (2007). Código de Faltas de la Provincia de Córdoba. Texto Ordenado 2007. Acceso el 25 de febrero, 2013, en http://web2.cba.gov.ar/web/leyes.nsf/0/ E0BD40709BEAA1DD8325740B005296D9

Lozano Gerena, L. C., Castro Guerrero, A. M., \& Moreno, J. H. (2008). Estilos atribucionales sobre la violencia de pareja de un grupo de mujeres maltratadas de la ciudad de Bogotá. Psicogente, 11(20), 122-133.

Manzi, J., Ruiz, S., Krause, M., \& Meneses, A. (2004). Memoria colectiva del golpe de Estado de 1973 en Chile. Interamerican Journal of Psychology, 38(2), 153-169.

Marrero, R., Moraza Pulla, O., Matud Aznar, M. P., Carballeira Abella, M., \& Aguilera Avila, L. (2003). Mujeres maltratadas por sus parejas. Atribuciones causales y dinámica de la violencia. Clepsydra: Revista de Estudios de Género y Teoría Feminista, 2, 57-70.

McCluskey, J., Terrill, W., \& Paoline, E. (2005). Peer group aggressiveness and the use of coerción in police-suspect encounters. Police Practice and Research, 6(1), 19-37.

Mendizábal, N. (2006). Los componentes del diseño flexible en la investigación cualitativa. In I. Vasilachis de Gialdino (Comp.), Estrategias de investigación cualitativa (pp. 65106). Barcelona: Gedisa.

Monjardet, D. (2010). Lo que hace la policía: sociología de la fuerza pública. Buenos Aires: Prometeo Libros.

Pinto Mascareño, R. (2010). Los movimientos sociales y los marcos de acción colectiva que apoyan la lucha contra la precariedad laboral. Tesis Doctoral, Universidad Complutense de Madrid.

Pronin, E., Kennedy, K., \& Butsch, S. (2006). Bombing versus negotiating: How preferences for combating terrorism are affected by perceived terrorist rationality. Basic and Applied Social Psychology, 28(4), 385-392.

Richaud de Minzi, M. C. (2004). Subjective and objective causality in science: A topic for attribution theory? Interdisciplinaria, n. spe., 149-159.

Sabucedo, J. M., Blanco, A., \& De la Corte, L. (2003). Beliefs which legitimize political violence against the innocent. Psicothema, 15(4), 550-555.

Sirimarco, M. (2010). Introducción. In M. Sirimarco (Comp.), Estudiar la policía: la mirada de las ciencias sociales sobre la institución policial (pp. 9-25). Buenos Aires: Teseo.
Suárez de Garay, M. (2005). Armados, enrejados, desconfiados... Tres breves lecturas sobre la cultura policial mexicana. Política y Sociedad, 42(3), 87-102.

Terrill, W. \& Mastrofski, S. (2002). Situational and officerbased determinants of police coercion. Justice Quarterly: $J Q, 19(2), 215$ - 249.

Terrill, W., Paoline, E., \& Manning, P. (2003). Police culture and coercion. Criminology, 41(4), 1003-1034.

Vásquez Cabrera, J. J. (2008). Factores en las atribuciones causales del terrorismo islamista. Psicología Política, 36, 77-93.

Vizdome-Lozano, J. C. \& Luciano, C. (2006). Locus de Control y autorregulación conductual: revisiones conceptual y experimental. International Journal of Clinical and Health Psychology, 6(3), 729-751.

Worden, R. E. (1995). Police officers' beliefs systems. A framework for analysis. American Journal of Police, 14(1), 49-81.

Yñiguez Navas, A. (2007). Reglas básicas de la práctica policial y funcionamiento interno de la Policía. Cuadernos de Trabajo Social, 20, 57-73.

Submissão em: 10/10/2013

Aceite em: 23/03/2014

Julián Martínez es Investigador asociado en la Facultad Latinoamericana de Ciencias Sociales (FLACSO - Sede Ecuador). Licenciado en Psicología por la Universidad Nacional de Córdoba. Máster en Ciencias Políticas por FLACSO - Ecuador. Exbecario de Postgrado en FLACSO -Ecuador. Dirección: Francisco Montalvo Oe9316 y Bella Aurora. CP 170132. Quito, Ecuador. E-mail: julian.martinez.r@gmail.com

Patricia Mariel Sorribas es Docente de la Facultad de Psicología - Universidad Nacional de Córdoba - y Facultad de Ciencia Política y Relaciones Internacionales - Universidad Católica de Córdoba, Argentina. Doctora en Psicología (Universidad Nacional de Córdoba). Exbecaria de postgrado (CONICET, SeCyT/UNC, Fundación Carolina, Red Macrouniversidades, AECID). Miembro del Programa "Democracia y Ciudadanía en Sudamérica" del Centro de Estudios Avanzados de la Universidad Nacional de Córdoba. E-mail: pamsorribas@yahoo.com.ar

\section{Como citar:}

Martinez, J. \& Sorribas, P. M. (2014). Atribuciones sobre el uso de la fuerza policial desde la perspectiva del agente. Psicologia \& Sociedade, 26(2), 430-439. 